\title{
Impact of Neoadjuvant Chemotherapy on the Rate of Bowel Resection in Advanced Epithelial Ovarian Cancer
}

\author{
CHARLES-ANDRÉ PHILIP ${ }^{1,2}$, AURÉLIE PELISSIER ${ }^{1,3}$, CLAIRE BONNEAU $^{1}$, \\ DELPHINE HEQUET $^{1}$, ROMAN ROUZIER ${ }^{1,3}$ and NICOLAS POUGET ${ }^{1}$ \\ ${ }^{1}$ Department of Breast and Gynecological Surgery, René Huguenin Centre, Curie Institute, Saint Cloud, France; \\ ${ }^{2}$ Medical School, Claude Bernard Lyon 1 University, Villerbanne, France; \\ ${ }^{3}$ EA 7285: Clinical Risks and Security in Women's Health and Perinatal Health, \\ Versailles-St-Quentin-en-Yvelines University, St-Quentin-en-Yvelines, France
}

\begin{abstract}
Aim: To assess the decrease in the number of bowel resections $(B R)$ necessary to achieve complete cytoreduction (CC-O) in advanced epithelial ovarian cancer (EOC) permitted by neoadjuvant chemotherapy (NAC). Patients and Methods: Patients were selected from a population of advanced EOC cases diagnosed between 2002 and 2009 at the Curie Institute: 97 patients with Federation International of Gynecology and Obstetrics IIIc and IV with unresectable disease treated with NAC followed by interval debulking surgery were included. We proceeded to a systematic blinded review of all the surgical reports pre-and post-NAC by two different surgeons to assess the surgical procedures required to obtain CC-O. Results: Before NAC, at least 84 patients ( $87 \%)$ would have required BR to obtain a CC-O resection. At interval debulking surgery, 47 (49\%) still required a BR, which corresponds to a decrease of $38 \%$ $(p<0.0001)$. The same decrease was observed for resection of small bowel, colon and rectosigmoid, as follows: 54 to 17 (77\% to $24 \%, p<0.0001)$, 45 to $19(56 \%$ to $24 \%, p<0.0001)$ and 72 to $25(90 \%$ to $31 \%, p<0.0001)$, respectively. The median overall survival (OS) among CC-O patients with and without BR was 57 months $[95 \%$ confidence interval $(C I)=25-90$ months] and 50 months $[95 \% \quad C I=43-57$ months], respectively $(p=0.71)$. The OS among patients without complete resection was significantly worse, with a median of 21 months (95\% CI=17-32 months, $p<0.0001$ ). Conclusion: NAC significantly reduces the need and rate of
\end{abstract}

Correspondence to: Aurélie Pelissier, Department of Breast and Gynecological Surgery, Centre René Huguenin, Institut Curie, 35 rue Dailly, 92210 Saint Cloud, France. Tel: +33 147111521, Fax: +33 147111522, e-mail: a.komorek@gmail.com

Key Words: Advanced epithelial ovarian cancer, neoadjuvant chemotherapy, bowel resection
$B R$ in advanced EOC, but also of small bowel, colon and rectosigmoid resection. There is no loss of $O S$, after $B R$ especially if the debulking surgery is complete.

Ovarian cancer is the seventh most common cancer in women with a worldwide incidence of 239,000 cases in 2012 and is the worst gynecological cancer in terms of prognosis with a mortality rate of $63.6 \%$ (1). The majority of women with ovarian cancer are diagnosed at advanced stages of the disease explaining this poor prognosis and justifying the use of extensive cytoreductive surgery (2).

The standard treatment in Federation International of Gynecology and Obstetrics (FIGO) stage IIIC and IV ovarian cancer is primary cytoreductive surgery (PCS) followed by platinum-based adjuvant chemotherapy (3). This standard of care is controversial since the 2010 publication of the pivotal phase III study by the European Organization for Research and Treatment of Cancer (EORTC) by Vergote et al. which showed that patients receiving neoadjuvant chemotherapy (NAC) followed by interval cytoreductive surgery (ICS) have similar outcomes to those undergoing PCS in terms of survival (4). A second published trial (MRC CHORUS trialCRUK 07/009) reached the same conclusion, and therefore it is now widely supported to use NAC when surgical debulking seems unfeasible (5).

The most important prognostic factor, independent of the delay of surgery and chemotherapy, is the size of the residual disease. Survival is better when resection is complete, which has been demonstrated both for PCS and NAC combined with $\operatorname{ICS}(4,6)$.

The digestive tract is frequently involved in advanced ovarian cancer (AOC), which justifies bowel resection (BR) in 60 to $70 \%$ of patients when a complete PCS is feasible (7$9)$. The major site of resection is the recto-sigmoid (48-55\%), followed by the rest of the colon (18-20\%) and the small bowel (6-27\%) depending on the series (7-9). Although some 
series report a lower rate of BR after NAC from 27-49\% (1011 ), there is no published study specifically evaluating the need for BR prior to and after NAC.

The aim of this study was to evaluate the need for BR to obtain cyoreduction prior to and after NAC in a population of patients with AOC patients treated by NAC, and to determine which was more effective using each patient as its own control case. We also describe the impact on the survival rate according to the necessity of BR and the level of residual disease.

\section{Patients and Methods}

Patient selection. We conducted a retrospective study using the Curie Institute database. Patients with advanced epithelial ovarian cancer stage IIIC and IV of the FIGO classification were included if they had disease that was considered unresectable by the surgeon during primary surgical evaluation and if they had received platinum-based NAC between January 2002 and December 2009. The study was approved by the Institutional Review Board of Curie Institute (approval number: 2013-10).

A histological diagnosis of epithelial ovarian carcinoma was mandatory. The inclusion criteria were a laparotomy or laparoscopy exploration with minimal surgery (biopsies/simple adnexectomy) prior to 3-6 courses of platinum- and taxane-based NAC followed by ICS. Patients without an initial surgical evaluation or with incomplete primary surgery including hysterectomy, lymphadenectomy or BR were excluded. When no surgical report was found in the database for primary evaluation or interval debulking surgery, the patient was also excluded. Clinical, biochemical, radiological and pathological variables were reviewed for each patient: age, weight, personal and family history, characteristics of the disease (histology, stage) and outcome.

Systematic review of the surgical data. We proceeded to a systematic review of all the surgical reports pre- and post-NAC performed by two different surgeons (CAP and NP) who did not participate in the surgeries. The two reports were anonymized, analyzed separately and each surgeon was initially blinded to the results of his colleague. The bowel locations were separated into three segments: the small bowel; the right, transverse and left colon; and the recto-sigmoid. When one or several bulky metastases were described in one of these areas, resection was considered 'necessary', even if it implied multiple anastomoses or if the complete resection was impossible. When a segment was clearly described as "normal", the resection was considered 'unnecessary'. When the surgical report did not precisely detail the extension of the lesion in a segment, the term "not available' was used. When discord was noted between the two reviewers, the report was reassessed jointly with a third reviewer (RR), and a majority consensus was applied.

We also calculated the absolute difference of the rates of necessary BR as the rate of BR estimated initially minus the rate of BR estimated after NAC. We also calculated the relative decrease in the rate of necessary BR as the rate of BR estimated after NAC per rate of patients who underwent BR estimated initially. We evaluated these rates not only for the entire bowel but also for each segment (excluding unavailable descriptions).

Surgical and histological reports were used to assess the number of resections effectively performed at the interval surgery. A resection was considered 'complete' if there was no macroscopic residual disease at the end of the procedure and if at least a hysterectomy with bilateral oophorectomy, omentectomy, pelvic and para-aortic lymphadenectomy were performed. Residual disease after surgery was classified according to Sugarbaker criteria: $\mathrm{CC} 0=$ nil, $\mathrm{CC} 1 \leq 0.25 \mathrm{~cm}, \mathrm{CC} 2=0.25-2.5 \mathrm{~cm}$, and $\mathrm{CC} 3 \geq 2.5 \mathrm{~cm}$ (12).

Statistical analysis. All the statistical analyses were performed with the software R version 3.0.2 (R Core Team 2013 by the R Foundation for Statistical Computing, Vienna, Austria). The Wilcoxon-MannWhitney test was used for the analysis of the quantitative variables, and the chi ${ }^{2}$ test or Fisher's exact tests were used for the qualitative variables. Statistical analyses to test the effect of NAC on BR in general and location by location were conducted using the Mc Nemar test for paired series. The overall survival (OS) was estimated using the Kaplan-Meier method and compared using the log-rank test. The survival end point was the interval between the date of diagnosis and the date of issue (death, last consultation, or loss to follow-up).

\section{Results}

We identified 118 patients who met the inclusion criteria and had AOC that was initially deemed to be unresectable. We included 97 patients after the exclusion of 21 patients who underwent incomplete primary surgery $(n=17)$ or had no surgical report that sufficiently documented the case in the database $(n=4)$. The median follow-up was 39.4 months. The clinical, biological and pathological characteristics are summarized in Table I.

The mean age at diagnosis was 63.4 years. The histological findings were dominated by serous adenocarcinoma (95\%) and grade III tumors (63\%); $71 \%$ were FIGO stage IIIC and $29 \%$ were stage IV. The mean number of NAC courses was 5.9 , and a complete cytoreduction was achieved in 64 patients (66\%). There were no significant differences between the characteristics of the group of patients who underwent BR $(n=26)$ and those of the group who did not $(n=71)$.

The requirement for BR during the initial evaluation and at the interval surgery is reported in Figure 1. Agreement between the reviewers in terms of BR requirement was $98 \%$. In the primary surgical assessment, at least one BR seemed to be necessary in 86 patients (89\%) and unnecessary in 11 patients $(11 \%)$. At the interval surgery, a BR seemed necessary and unnecessary in $50(52 \%)$ and $47(48 \%)$ patients, respectively. This corresponds to a $36 \%$ absolute decrease in BR $(p<0.0001)$. A BR was effectively performed in 26 patients. CC- 0 was obtained in 20/26 (77\%) patients when a BR was performed and in 43/71 (61\%) cases when BR was not (Figure 1). In 24 patients with bowel involvement at the interval surgery (48\%), no BR was performed because disease remained unresectable.

When focusing on the 86 patients with a theoretical BR requirement prior to NAC, a BR was still necessary in 48 cases $(56 \%)$ after NAC, whereas it was no longer necessary in 38 patients, which corresponds to a $42 \%$ relative decrease in the theoretical requirement for BR. A BR was effectively performed in 24 patients (28\%) and resulted in a complete 


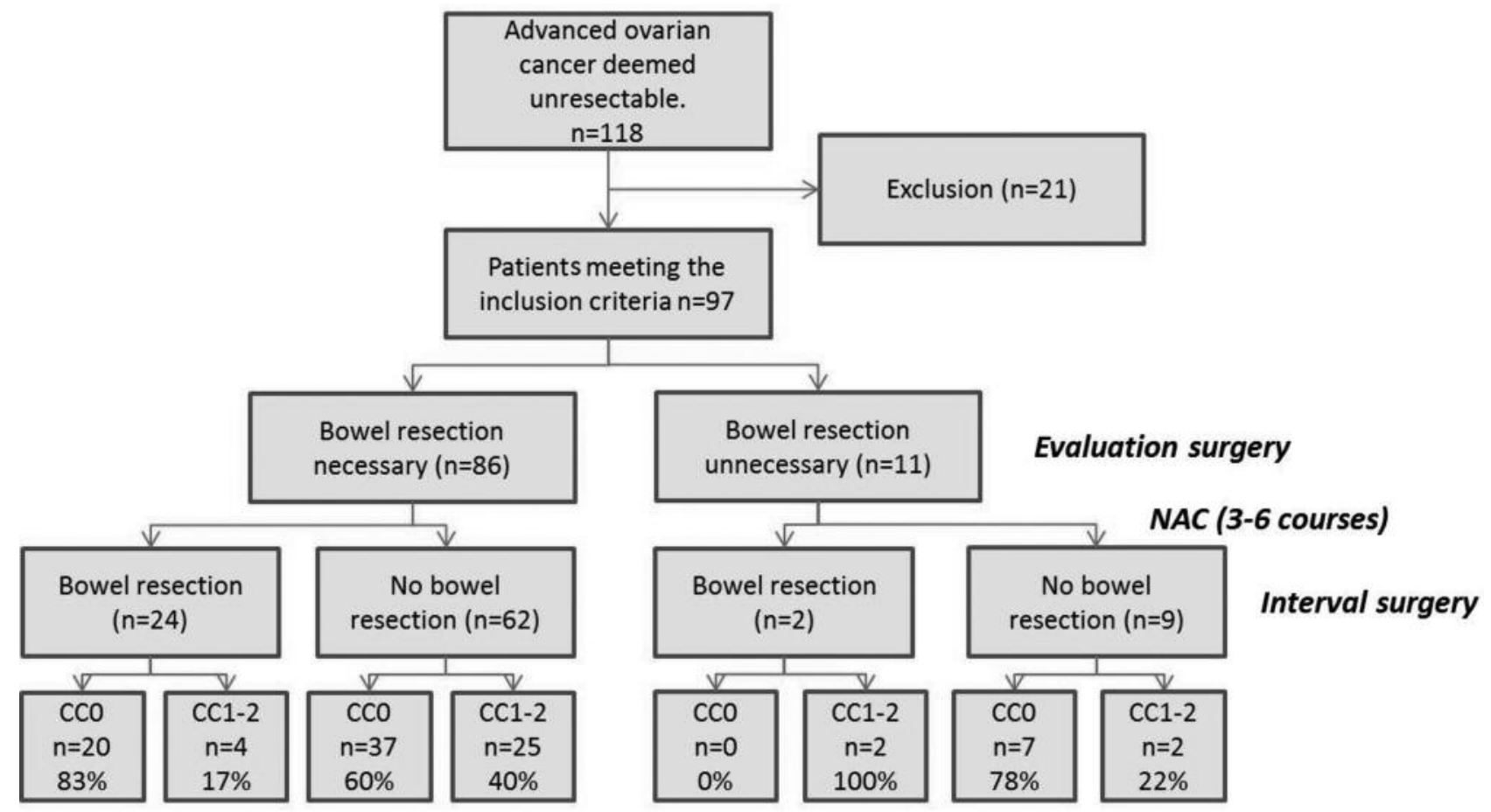

Figure 1. Flow chart. CCO: Non-residual disease after interval debulking surgery (IDS); CC1+: residual disease after IDS (CC1 $\leq 0.25 \mathrm{~cm}$; $C C 2=0.25-2.5 \mathrm{~cm} ; C C 3 \geq 2.5 \mathrm{~cm}) ;$ NAC: neoadjuvant chemotherapy.

Table I. Patient and tumor characteristics.

\begin{tabular}{|c|c|c|c|c|}
\hline Characteristic & Overall population $(n=97)$ & Bowel resection $(n=26)$ & No bowel resection $(\mathrm{n}=71)$ & $p$-Value \\
\hline Mean age (SD), years & $63.4(9.4)$ & $63.1 \pm 5.8$ & $63.5 \pm 10.44$ & 0.78 \\
\hline Mean BMI (SD), $\mathrm{kg} / \mathrm{m}^{2}$ & $23.6(3.8)$ & 24.2 & 23.3 & 0.39 \\
\hline Family history of cancer, $\mathrm{n}(\%)$ & $43(45 \%)$ & $12(46 \%)$ & $31(44 \%)$ & 0.71 \\
\hline Personal history of cancer, $\mathrm{n}(\%)$ & $17(18 \%)$ & $5(19 \%)$ & $12(17 \%)$ & 0.79 \\
\hline \multicolumn{5}{|l|}{ Histology (adenocarcinoma), n (\%) } \\
\hline Serous & $95 \%$ & $26(100 \%)$ & $66(93 \%)$ & - \\
\hline Mucinous & $2(2 \%)$ & - & $2(3 \%)$ & - \\
\hline Endometrioid & $2(2 \%)$ & - & $2(3 \%)$ & - \\
\hline Clear cell & $1(1 \%)$ & - & $1(1 \%)$ & - \\
\hline \multicolumn{5}{|l|}{ Grade, n (\%) } \\
\hline NA & 10 & 2 & 8 & \\
\hline I & $4(5 \%)$ & $2(8 \%)$ & $2(3 \%)$ & 0.41 \\
\hline II & $30(35 \%)$ & $9(38 \%)$ & $21(33.3 \%)$ & 0.35 \\
\hline III & $5363 \%)$ & $13(54 \%)$ & $40(63.5 \%$ & 0.44 \\
\hline FIGO stage, n (\%) & & & & 0.62 \\
\hline IIIc & $69(71 \%)$ & $17(65 \%)$ & $52(73 \%)$ & - \\
\hline IV & $2829 \%$ ) & $6(35 \%)$ & $19(27 \%)$ & - \\
\hline Mean pre-NAC CA-125 (SD), U/ml & $2197(3361)$ & $2438(3723)$ & 0.13 & \\
\hline Mean no. of cycles of NAC (SD) & $5.9(1.4)$ & $5.69(1.5)$ & $6.04(1.4)$ & 0.31 \\
\hline \multicolumn{5}{|l|}{ Cytoreduction (interval surgery), n (\%) } \\
\hline $\mathrm{CC} 0$ & $64(66 \%)$ & $20(77 \%)$ & $44(62 \%)$ & \\
\hline $\mathrm{CC} 1-2$ & $33(34 \%)$ & $6(23 \%)$ & $27(38 \%)$ & 0.12 \\
\hline
\end{tabular}

SD, Standard deviation; BMI, body mass index; HRT, hormone replacement therapy; FIGO, International Federation of Gynecology and Obstetrics; NAC, neoadjuvant chemotherapy. 
Table II. Rate of bowel resection before and after neoadjuvant chemotherapy (NAC).

\begin{tabular}{|c|c|c|c|c|c|c|}
\hline \multirow[t]{2}{*}{ Before NAC } & \multirow[b]{2}{*}{$\mathrm{n}(\%)$} & \multicolumn{3}{|c|}{ After NAC, n (\%) } & \multirow[b]{2}{*}{ Relative decrease } & \multirow[b]{2}{*}{ Absolute decrease } \\
\hline & & Necessary & Unnecessary & $p$-Value & & \\
\hline Bowel resection & & & & $<0.0001$ & & \\
\hline Necessary & $86(89)$ & $48(56)$ & $38(44)$ & & $42 \%$ & $37 \%$ \\
\hline Unnecessary & $11(11)$ & $2(18)$ & $9(82)$ & & & \\
\hline Total & 97 & $50(52)$ & $47(48)$ & & & \\
\hline Small bowel resection & & & & $<0.0001$ & & \\
\hline Necessary & $54(77)$ & $17(32)$ & $37(69)$ & & $65 \%$ & $50 \%$ \\
\hline Unnecessary & $16(23)$ & $2(13)$ & $14(88)$ & & & \\
\hline Total & 70 & $19(27)$ & $51(73)$ & & & \\
\hline Not evaluable & 27 & & & & & \\
\hline Colon resection & & & & $<0.0001$ & & \\
\hline Necessary & $45(56)$ & $19(42)$ & $26(58)$ & & $51 \%$ & $29 \%$ \\
\hline Unnecessary & $35(44)$ & $3(9)$ & $32(91)$ & & & \\
\hline Total & 80 & $22(28)$ & $58(72)$ & & & \\
\hline Not evaluable & 17 & & & & & \\
\hline Rectosigmoid resection & & & & $<0.0001$ & & \\
\hline Necessary & $72(90)$ & $25(35)$ & $47(65)$ & & $63 \%$ & $56 \%$ \\
\hline Unnecessary & $8(10)$ & $2(25)$ & $6(75)$ & & & \\
\hline Total & 80 & $27(34)$ & $53(66)$ & & & \\
\hline Not evaluable & 17 & & & & & \\
\hline
\end{tabular}

NAC: Neoadjuvant chemotherapy; n: number of patients; $\mathrm{CC} 0$ : no gross residuals; $\mathrm{CC}>0$ : macroscopic residuals.

resection in 20 cases $(83 \%)$. In 62 patients, no BR was performed, and a complete resection was achieved in 37 cases $(60 \%)$. Conversion from BR "needed at initial evaluation" to "not necessary" at interval surgery resulted in complete resection without $\mathrm{BR}$ in $37 / 86$ patients $(43 \%)$. The $\mathrm{CC}-0$ rate was higher when BR was not necessary after NAC $(p<0.0001)$.

Conversely, in the 11 patients with no requirement for BR initially, a BR was performed in two cases (18\%) at interval debulking surgery most likely because of involvement not observed during the initial evaluation. These two patients underwent incomplete surgery because of bulky disease.

When considering the 64 patients who underwent a complete resection at interval surgery, a BR had been performed in 20 patients $(31 \%)$ compared to 57 patients at the initial evaluation ( $89 \%$ ), corresponding to a $58 \%$ absolute decrease as a benefit of NAC.

In Table II, we describe the bowel segment involvement that required resection at the initial evaluation and at interval surgery. The descriptions in operative reports were insufficient in 27 cases for small bowel disease and in 17 cases for large bowel disease. Fifty-nine patients $(60 \%)$ had two or more involved bowel segments. NAC reduced the necessity for resection of the small bowel, recto-sigmoid, and the rest of the colon in $50 \%, 29 \%$, and $56 \%$, respectively (absolute reduction rate).

The global median OS of our population was 40 months [95\% confidence interva $(\mathrm{CI})=36$ to 49 months] from time of diagnosis (Figure 2A). The median OS of patients who underwent complete resection (57 months) was significantly higher than that of patients who underwent incomplete resection (21 months) $(p<0.0001)$ (Figure 2B).

There were no significant differences in survival between patients who required $\mathrm{BR}$ and those who did not during the initial evaluation (median $\mathrm{OS}=39$ vs. 52 months, $p=0.46$ ) (Figure 2C) The median OS was significantly worse in patients who still theoretically required a BR after NAC based on univariate analysis (median $\mathrm{OS}=23.8 v s .49 .9$ months, $p=0.0007$ ).

When considering only patients in whom complete resection was achieved, there was no significant difference between patients who required $\mathrm{BR}$ or not prior to NAC (median OS=57.4 vs. 63.3 months, $p=0.81$ ) and after NAC (median $\mathrm{OS}=57.4$ vs. 49.9 months, $p=0.75$ ) (figures not shown). There was also no significant difference in survival whether a BR was effectively achieved or not in these patients (median OS=57.4 vs. 49.9 months, $p=0.71$ ) (Figure 2D). When considering patients in whom complete resection was not achieved, there was also no difference in survival between the ones who underwent effective BR and those who did not (median OS=29.5 vs. 21.4 months, $p=0.58$ ) (Figure 2D).

\section{Discussion}

This study shows that platinum-based NAC for AOC reduces the number of patients who need BR by $37 \%$ (absolute reduction) $(p<0.0001)$. Similar reduction rates are observed for all the digestive segments, with a relative reduction of 

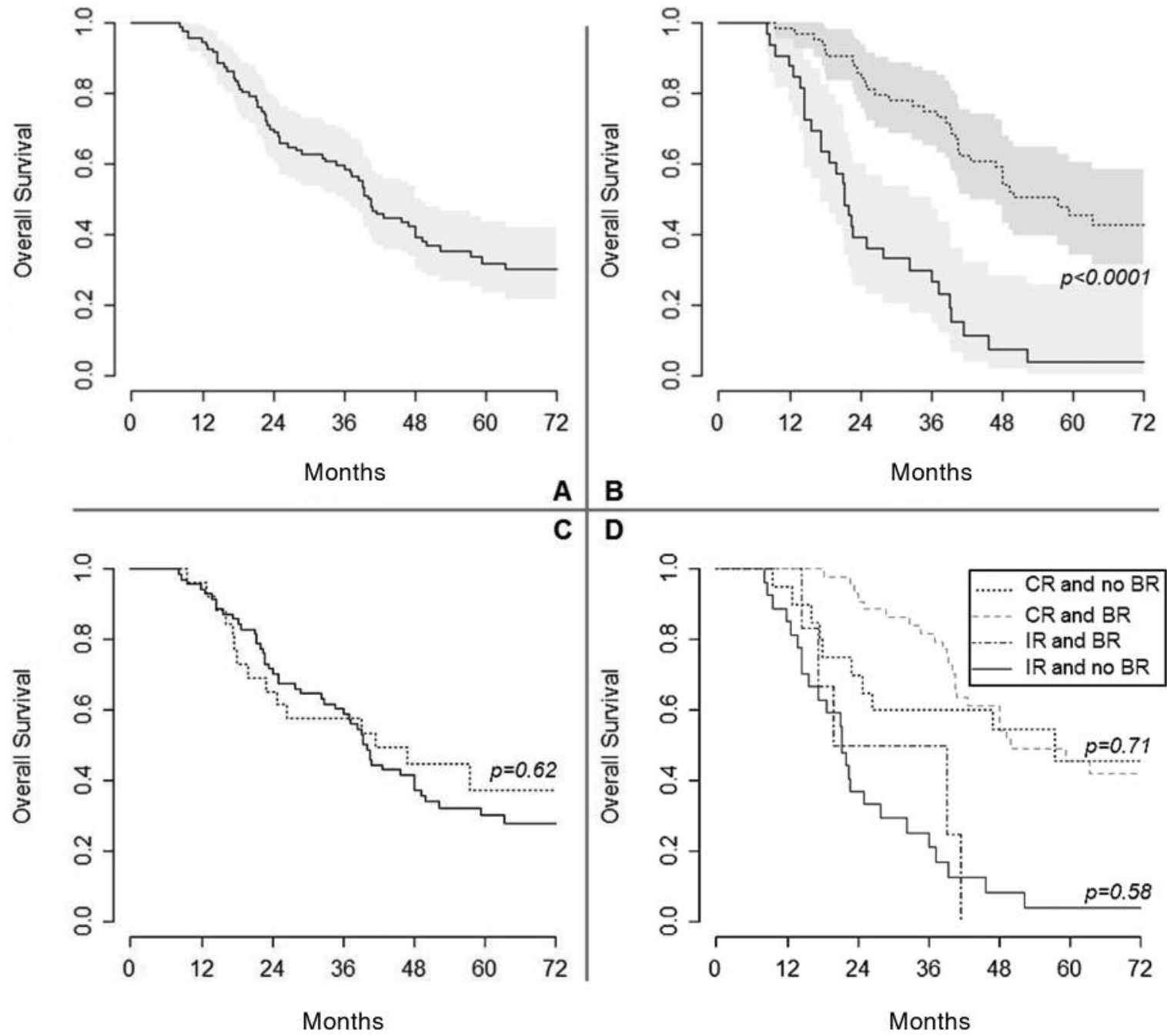

Figure 2. Overall survival for the whole patient cohort (A), according to complete resection (CR, dotted line) or incomplete resection (IR, unbroken line) (B), according to global bowel resection performed (BR, dotted line) or not (unbroken line) (C), and according to BR and CR (D).

resection in small-bowel, recto-sigmoid and the rest of the colon of $50 \%, 56 \%$ and $29 \%$, respectively $(p<0.0001)$. The reduction in the need for $\mathrm{BR}$ resulted in more complete cytoreductions. Interestingly, there was no significant impact on OS in patients who required a BR prior to NAC $(p=0.46)$, nor in those who underwent BR after NAC $(p=0.62)$. The only significant prognostic factor after NAC remained the possibility of a complete resection $(p<0.0001)$. NAC combined with ICS is, therefore, an effective alternative to PCS when the possibility of a complete resection is initially uncertain and should be considered when the number of necessary BR threatens the functional prognosis of the patient. Patients who underwent BR at ICS exhibited similar survival to patients who did not when stratified based on complete resection status.
Some potential bias should be considered prior to interpretation of the results of this study. Firstly, this was a retrospective study over a relatively long period. The resectability criteria were not pre-determined, and the surgeon made the decisions according to their experience and each patient's general status. We observed important changes during the study period with an increasing number of radical surgeries over time, including diaphragmatic peritonectomy, partial pancreatectomy and splenectomy, which was associated with an improved rate of complete resections. However, involvement of the bowel and the effect of NAC are measurable independently of the final surgical procedure. The use of surgical reports to assess the necessity of BR is also questionable as it can lead to substantial errors if some segments are not well described. A BR was performed at 
interval debulking surgery in two out of the 11 patients who did not require BR initially. This suggests that some lesions, however infrequently, are not observed during the initial evaluation. To optimize the evaluation of surgical reports, we used a double-blinded review process, which produced the same decision by the two different surgeons in most cases. This procedure allowed us to create a case-control study in which each patient was their own control. We also acknowledge that most patients received more than four courses of chemotherapy, which is more than typically administered. However, our patients had very advanced disease, as we focused only on patient with unresectable disease at the time of diagnosis. This also explains the relatively low number of patients in this study. We investigated but did not find (data not shown) any correlation between bowel clearance and the number of courses of chemotherapy administered.

The aim of this study was not to compare the performance of PCS to NAC in AOC, as we agree that patients should undergo PCS when a complete resection seems feasible without a major risk in terms of surgical morbidity and mortality (13-14). However, the criteria that define unresectable disease are still debated $(9,15)$. Extensive lesions of the small bowel, lesions $>2 \mathrm{~cm}$ of the mesentery and the need for more than two intestinal sutures are some of the criteria that have been reported to be frequently associated with incomplete resection or with increased postoperative complications $(7,9,14,16-19)$. We performed this study to assess how many BRs can be spared and the rate of complete resection that can be achieved with NAC in these cases. Regarding the small bowel lesions, which were usually associated with mesenteric extensions, we showed a $68.5 \%$ global reduction in the need for resection. When focusing on patients in whom complete resection was achieved, there was only a $6.4 \%$ resection of this segment. This most likely reflects that the necessity for multiple small BRs is probably one of the most limiting factors for complete resection. These results are consistent with the above criteria in primary surgery. The resection of the recto-sigmoid and the rest of the colon were not specifically a limiting factor for complete resection, unless multiple intestinal sutures were required.

The rate of patients achieving a complete resection in this study was $66 \%$. This result is also consistent with published data on NAC with a complete resection rate ranging from 47 $73 \%(4,10,14,20-21)$. As previously reported, this important number of patients without any residual disease after NAC and ICS is superior to the typical results of primary surgery (6). However, the need for BR after NAC, particularly if associated with a small bowel extension, was associated with a higher rate of residual disease and therefore with poorer prognosis. The survival outcomes of the population of our study are interesting showing a global median OS of 40.4 months. These results are similar to the higher median OS published in ICS following NAC, which usually ranges from
$23-53 \%(10,13,20-27)$. When focusing on patients achieving complete resection, there was a median OS of 57.4 months, which is only slightly lower than outcomes reported in PCS publications with median OS of approximately 69 months (28). The need for BR did not significantly reduce patient survival, as has been reported in primary surgery (7-9), thus advocating for cytoreductive surgery after NAC. Nevertheless, the $\mathrm{CC}-0$ rate was higher when BR was not necessary after NAC $(p<0.0001)$. As BR is associated with higher morbidity, our study favors the use of NAC when multiple BRs are necessary to achieve $\mathrm{CC}-0$ based on the initial evaluation.

In conclusion, platinum and taxane-based NAC followed by ICS significantly reduces the rate of overall digestive surgical resection as well as that of small bowel, colon and rectosigmoid resection. Bowel resection should be performed after NAC to obtain complete cytoreduction and positively impact outcome.

\section{Conflicts of Interest}

None.

\section{Acknowledgements}

The present study was supported by the "Fondation pour la Recherche Médicale” (FRM).

\section{References}

1 Ferlay J, Soerjomataram I, Dikshit R, Eser S, Mathers C, Rebelo M, Parkin DM, Forman D and Bray F: Cancer incidence and mortality worldwide: sources, methods and major patterns in GLOBOCAN 2012. Int J Cancer 136(5): E359-386, 2015.

2 Holschneider $\mathrm{CH}$ and Berek JS: Ovarian cancer: epidemiology, biology, and prognostic factors. Semin Surg Oncol 19(1): 3-10, 2000.

3 Du Bois A, Quinn M, Thigpen T, Vermorken J, Avall-Lundqvist E, Bookman M, Bowtell D, Brady M, Casado A, Cervantes A, Eisenhauer E, Friedlaender M, Fujiwara K, Grenman S, Guastalla JP, Harper P, Hogberg T, Kaye S, Kitchener H, Kristensen G, Mannel R, Meier W, Miller B, Neijt JP, Oza A, Ozols R, Parmar M, Pecorelli S, Pfisterer J, Poveda A, Provencher D, Pujade-Lauraine E, Randall M, Rochon J, Rustin G, Sagae S, Stehman F, Stuart G, Trimble E, Vasey P, Vergote I, Verheijen R, Wagner U; Gynecologic Cancer Intergroup; AGOOVAR; ANZGOG; EORTC; GEICO; GINECO; GOG; JGOG; MRC/NCRI; NCIC-CTG; NCI-US; NSGO; RTOG; SGCTG; IGCS; Organizational team of the two prior International OCCC: 2004 consensus statements on the management of ovarian cancer: final document of the 3rd International Gynecologic Cancer Intergroup Ovarian Cancer Consensus Conference (GCIG OCCC 2004). Ann Oncol 16(Suppl 8): viii7-viii12, 2005.

4 Vergote I, Trope CG, Amant F, Kristensen GB, Ehlen T, Johnson $\mathrm{N}$, Verheijen RH, van der Burg ME, Lacave AJ, Panici PB, Kenter GG, Casado A, Mendiola C, Coens C, Verleye L, Stuart GC, Pecorelli S, Reed NS; European Organization for Research and Treatment of Cancer-Gynaecological Cancer Group; NCIC Clinical Trials Group: Neoadjuvant chemotherapy or primary 
surgery in stage IIIC or IV ovarian cancer. N Engl J Med 363(10): 943-953, 2010.

5 Kehoe S, Hook J, Nankivell M, Jayson GC, Kitchener HC, Lopes T, Luesley D, Perren T, Bannoo S, Mascarenhas M, Dobbs S, Essapen S, Twigg J, Herod J, McCluggage G, Parmar $\mathrm{M}$ and Swart AM: Primary chemotherapy versus primary surgery for newly diagnosed advanced ovarian cancer (CHORUS): an open-label, randomised, controlled, non-inferiority trial. Lancet 386: 249-250, 2015.

6 Chang SJ, Hodeib M, Chang J and Bristow RE: Survival impact of complete cytoreduction to no gross residual disease for advanced-stage ovarian cancer: a meta-analysis. Gynecol Oncol 130(3): 493-498, 2013.

7 Eisenkop SM and Spirtos NM: Procedures required to accomplish complete cytoreduction of ovarian cancer: is there a correlation with "biological aggressiveness" and survival? Gynecol Oncol 82(3): 435-441, 2001.

8 Eisenhauer EL, Abu-Rustum NR, Sonoda Y, Levine DA, Poynor EA, Aghajanian C, Jarnagin WR, DeMatteo RP, D'Angelica MI, Barakat RR and Chi DS: The addition of extensive upper abdominal surgery to achieve optimal cytoreduction improves survival in patients with stages IIIC-IV epithelial ovarian cancer. Gynecol Oncol 103(3): 1083-1090, 2006.

9 Salani R, Zahurak ML, Santillan A, Giuntoli RL, 2nd and Bristow RE: Survival impact of multiple bowel resections in patients undergoing primary cytoreductive surgery for advanced ovarian cancer: a case-control study. Gynecol oncol 107(3): 495-499, 2007.

10 Stoeckle E, Bourdarias L, Guyon F, Croce S, Brouste V, Thomas $\mathrm{L}$ and Floquet A: Progress in survival outcomes in patients with advanced ovarian cancer treated by neo-adjuvant platinum/taxane-based chemotherapy and late interval debulking surgery. Ann Surg Oncol 21(2): 629-636, 2014.

11 Colombo PE, Mourregot A, Fabbro M, Gutowski M, Saint-Aubert B, Quenet F, Gourgou S and Rouanet P: Aggressive surgical strategies in advanced ovarian cancer: a monocentric study of 203 stage IIIC and IV patients. Eur J Surg Oncol 35(2): 135-143, 2009.

12 Jacquet $\mathrm{P}$ and Sugarbaker PH: Clinical research methodologies in diagnosis and staging of patients with peritoneal carcinomatosis. In: Peritoneal Carcinomatosis: Principles of Management. Sugarbaker PH (ed.) Boston: Kluwer, 1996.

13 Bristow RE, Eisenhauer EL, Santillan A and Chi DS: Delaying the primary surgical effort for advanced ovarian cancer: a systematic review of neoadjuvant chemotherapy and interval cytoreduction. Gynecol Oncol 104(2): 480-490, 2007.

14 Brun JL, Rouzier R, Selle F, Houry S, Uzan S and Darai E: Neoadjuvant chemotherapy or primary surgery for stage III/IV ovarian cancer: contribution of diagnostic laparoscopy. BMC Cancer 9: 171, 2009.

15 Fotopoulou C, Richter R, Braicu EI, Schmidt SC, Lichtenegger $\mathrm{W}$ and Sehouli J: Can complete tumor resection be predicted in advanced primary epithelial ovarian cancer? A systematic evaluation of 360 consecutive patients. Eur J Surg Oncol 36(12): 1202-1210, 2010.

16 Bristow RE, Duska LR, Lambrou NC, Fishman EK, O’Neill MJ, Trimble EL and Montz FJ: A model for predicting surgical outcome in patients with advanced ovarian carcinoma using computed tomography. Cancer 89(7): 1532-1540, 2000.

17 Aletti GD, Dowdy SC, Podratz KC and Cliby WA: Relationship among surgical complexity, short-term morbidity, and overall survival in primary surgery for advanced ovarian cancer. Am J Obstet Gynecol 197(6): 676e1-7, 2007.
18 Chereau E, Ballester M, Selle F, Cortez A, Darai E and Rouzier $\mathrm{R}$ : Comparison of peritoneal carcinomatosis scoring methods in predicting resectability and prognosis in advanced ovarian cancer. Am J Obstet Gynecol 202(2): 178e1- e10, 2010.

19 Fagotti A, Ferrandina G, Fanfani F, Garganese G, Vizzielli G, Carone V, Salerno MG and Scambia G: Prospective validation of a laparoscopic predictive model for optimal cytoreduction in advanced ovarian carcinoma. Am J Obstet Gynecol 199(6): 642e1-6, 2008.

20 da Costa Miranda V, de Souza Fede AB, Dos Anjos CH, da Silva JR, Sanchez FB, da Silva Bessa LR, de Paula Carvalho J, Filho EA, de Freitas D and del Pilar Estevez Diz M: Neoadjuvant chemotherapy with six cycles of carboplatin and paclitaxel in advanced ovarian cancer patients unsuitable for primary surgery: Safety and effectiveness. Gynecol Oncol 132(2): 287-291, 2014.

21 Rosen B, Laframboise S, Ferguson S, Dodge J, Bernardini M, Murphy J, Segev Y, Sun P and Narod SA: The impacts of neoadjuvant chemotherapy and of debulking surgery on survival from advanced ovarian cancer. Gynecol Oncol 134(3): 462-467, 2014.

22 Lee SJ, Kim BG, Lee JW, Park CS, Lee JH and Bae DS: Preliminary results of neoadjuvant chemotherapy with paclitaxel and cisplatin in patients with advanced epithelial ovarian cancer who are inadequate for optimum primary surgery. J Obstet Gynecol Res 32(1): 99-106, 2006.

23 Chan YM, Ng TY, Ngan HY and Wong LC: Quality of life in women treated with neoadjuvant chemotherapy for advanced ovarian cancer: a prospective longitudinal study. Gynecol Oncol 88(1): 9-16, 2003.

24 Fanfani F, Ferrandina G, Corrado G, Fagotti A, Zakut HV, Mancuso $S$ and Scambia G: Impact of interval debulking surgery on clinical outcome in primary unresectable FIGO stage IIIc ovarian cancer patients. Oncol 65(4): 316-322, 2003.

25 Steed H, Oza AM, Murphy J, Laframboise S, Lockwood G, DE Petrillo D, Sturgeon J and Rosen B: A retrospective analysis of neoadjuvant platinum-based chemotherapy versus up-front surgery in advanced ovarian cancer. Int $\mathrm{J}$ Gynecol Cancer 16(Suppl 1): 47-53, 2006.

26 Morice P, Dubernard G, Rey A, Atallah D, Pautier P, Pomel C, Lhommé C, Duvillard P and Castaigne D: Results of interval debulking surgery compared with primary debulking surgery in advanced stage ovarian cancer. J Am Coll Surg 197(6): 955-963, 2003.

27 Morice P, Brehier-Ollive D, Rey A, Atallah D, Lhomme C, Pautier P, Pomel C, Camatte S, Duvillard P and Castaigne D: Results of interval debulking surgery in advanced stage ovarian cancer: an exposed-non-exposed study. Ann Oncol 14(1): 74-77, 2003.

28 Chi DS, Musa F, Dao F, Zivanovic O, Sonoda Y, Leitao MM, Levine DA, Gardner GJ, Abu-Rustum NR and Barakat RR: An analysis of patients with bulky advanced stage ovarian, tubal, and peritoneal carcinoma treated with primary debulking surgery (PDS) during an identical time period as the randomized EORTC-NCIC trial of PDS vs. neoadjuvant chemotherapy (NACT). Gynecol Oncol 124(1): 10-14, 2012.

Received July 19, 2016

Revised August 14, 2016

Accepted August 18, 2016 